\title{
Article
}

\section{Premature ejaculation: therapist perspectives}

Brewer, Gayle and Tidy, Paul

Available at http://clok.uclan.ac.uk/15374/

Brewer, Gayle ORCID: 0000-0003-0690-4548 and Tidy, Paul (2016) Premature ejaculation: therapist perspectives. Sexual and Relationship Therapy . pp. 114. ISSN 1468-1994

It is advisable to refer to the publisher's version if you intend to cite from the work. http://dx.doi.org/10.1080/14681994.2016.1188200

For more information about UCLan's research in this area go to http://www.uclan.ac.uk/researchgroups/ and search for < name of research Group>.

For information about Research generally at UCLan please go to http://www.uclan.ac.uk/research/

All outputs in CLoK are protected by Intellectual Property Rights law, including Copyright law. Copyright, IPR and Moral Rights for the works on this site are retained by the individual authors and/or other copyright owners. Terms and conditions for use of this material are defined in the policies page.

\section{CLoK}

Central Lancashire online Knowledge www.clok.uclan.ac.uk

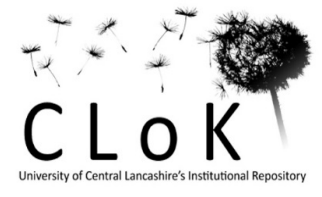




\title{
Premature Ejaculation: Therapist Perspectives
}

\author{
Gayle Brewer and Paul Tidy
}

Premature Ejaculation (PE) is a common sexual dysfunction affecting approximately 20-30\% of men (Porst, et al., 2007). Despite important issues relating to PE definition, diagnosis, and therapy, there is a paucity of research investigating the experiences of practitioners delivering PE treatment. For the present study, interviews were conducted with eight Psychosexual Therapists and subjected to Interpretive Phenomenological Analysis. Four master themes emerged from the analysis. These were Romantic Relationships, Vulnerability, Culture, and Diagnosis and Assessment. The Romantic Relationships theme included three sub-themes: Intimacy; Involvement; and Distress. Two sub-themes formed the Vulnerability theme: Self-Esteem; and Anxiety. The Culture theme contained three sub-themes: Religion and Ethnicity; Pornography; and Masculinity. The Diagnosis and Assessment theme included two sub-themes: Referral and Definitions. Findings have important implications for the diagnosis and treatment of PE. Future research should investigate therapist perceptions further, including those factors which impact on engagement with clients and PE treatment success.

Keywords: Interpretative Phenomenological Analysis; Interviews; Premature Ejaculation; Sexual Dysfunction; Therapy 


\section{Introduction}

Premature Ejaculation (PE) is a common sexual dysfunction affecting approximately $20-30 \%$ of men (Porst, et al., 2007). Though a range of measures have historically informed diagnosis (e.g. inability to delay ejaculation, intravaginal ejaculatory latency time, thrust number, and lack of voluntary control), PE has more recently been defined as "A persistent or recurrent pattern of ejaculation occurring during partnered sexual activity within approximately 1 minute following vaginal penetration and before the individual wishes it" (DSM-5, APA, 2013). The DSM-5 further specifies that this must have persisted for at least six months, cause significant distress, and occur on over $75 \%$ of occasions. According to the DSM-5, professionals diagnosing PE should also identify whether the condition is Lifelong, Acquired, Generalised, Situational, and Mild, Moderate or Severe.

Men with PE report increased anxiety (Porst, et al., 2007), emotional distress, and social phobia (Corretti, Pierucci, De Scisciolo, \& Nistita, 2006), together with decreased confidence (Symonds, Roblin, Hart, \& Althof, 2003) and quality of life (Rosen \& Althof, 2008). Relational quality of life is often negatively impacted by PE regardless of relationship status. Partnered men with PE report greater relationship difficulties (Sotomayor, 2005) and low sexual satisfaction (Rowland, Patrick, Rothman, \& Gagnon, 2007) whilst single men avoid new sexual relationships (Symonds, et al., 2003). Despite this, relatively few men report seeking treatment for PE and the majority of those receiving support experience little improvement (Porst, et al., 2007).

Therapist perspectives may reveal important information about the therapeutic relationship (Mackereth, Parekh, \& Donald, 2012), identify factors which facilitate or hinder treatment (Ackerman, \& Hilsenroth, 2003), and inform practice (Rybovic, Halkett, Banati, \& Cox, 2008). Therapist perspectives can also provide valuable information relating to the validity of 
condition classification (Kirschneck, Kirchberger, Amann, \& Cieza, 2011). Previous studies focusing on the experiences of psychosexual therapists are often focused on measurement issues (e.g. ejaculatory latency time, Corty \& Guardiani, 2008) or specific populations (e.g. lesbian patients, Hall, 2002) only. Therefore, the current study employed Interpretive Phenomenological Analysis to investigate therapist experiences of diagnosing and treating PE. The approach has been successfully employed with patients (Maguire, Stoddart, Flowers, McPhelim, \& Kearney, 2014), therapists (McCaffrey, 2013), and those delivering psychological interventions (Arvinen-Barrow, Penny, Hemmings, \& Corr, 2010).

\section{Method}

\section{Participants}

A purposive sample was recruited via email in October 2014. All participants $(N=8)$ were qualified Psychosexual Therapists practicing in the North West of England identified via the College of Sexual and Relationship Therapists (COSRT) website. Each participant had provided psychosexual therapy to men with PE for at least one year and was a registered member of a professional body such as the College of Sexual and Relationship Therapists (COSRT) or UK Council for Psychotherapy (UKCP). Participant age ranged from 45 to 63 years $(M=53.13, S D=7.66)$ and the majority of those recruited $(N=7)$ were female. Post qualified experience ranged from 8 to 27 years $(M=14.88, S D=5.91)$ and psychosexual therapeutic experience ranged from 4 to 20 years $(M=10.88, S D=6.31)$. Five therapists worked in both NHS settings and private practice and three worked in private practice only. See Table 1 for full participant details.

[Table 1 near here] 


\section{Materials and Procedure}

Prior to interview, participants completed a questionnaire identifying demographic status, professional training, and experience of clients with PE. Individual semi-structured interviews were then conducted at the participant's place of work, with only the interviewer (second author) and interviewee present. Each interview lasted between 40 and 90 minutes $(M=55.50, S D=17.57)$ resulting in 444 minutes of interview material. Open-ended questions prompted participants to narrate and reflect on their experiences. The interview schedule was prepared following appropriate guidelines (Smith, 1995) and questions were framed to obtain information about assessment and diagnosis, factors contributing to the development and maintenance of PE, treatment, and professional practice issues. Interviews were recorded on a portable hand-held device and transcribed verbatim. Interviews were anonymised at the point of transcription and subject to Interpretive Phenomenological Analysis (Smith, 1996). Informed written consent was obtained prior to participation and all participants agreed to the publication of direct quotations. Ethical approval was obtained from the host institution.

Interpretative Phenomenological Analysis provides a position and protocol for the analysis of experiential qualitative data. The method adopts phenomenological, hermeneutic, and idiographic perspectives (Smith, Flowers, \& Larkin, 2009) which seeks to describe, interpret, and understand the lived experience of a population and the manner in which individuals make sense of this lived experience. The researcher listened to the recordings and read the interview transcripts several times to aid familiarisation. Notes were made of significant areas of the text. Following further readings of the transcripts, notes were formed into emergent themes. The researcher then identified relationships between emerging themes and these were grouped into superordinate themes and sub-themes. The process was validated by discussions between the first and second author, during which the appropriateness of each theme and sub- 
theme was established. Principles proposed by Smith, et al. (2009) were adhered to throughout the data collection and analytic process to provide rigor and cohesion.

\section{Results}

Interpretative Phenomenological Analysis of interview transcripts generated four superordinate themes: (1) Romantic Relationships; (2) Vulnerability; (3) Culture; and (4) Diagnosis and Assessment. Each superordinate theme contained a number of sub-themes, as detailed in Table 2.

[Table 2 near here]

\section{Romantic Relationships}

\section{Intimacy}

Each therapist commented on the relationship between PE and sexual intimacy. They reported that men would often withdraw from their partner, avoiding both sexual and nonsexual intimacy. This withdrawal was commonly misinterpreted by their partner as a lack of sexual interest or an indication that the man was being unfaithful. The relationship difficulties were compounded by a lack of communication. Men that were not in a sexual relationship at the time of therapy often avoided initiating romantic relationships. One therapist suggested that men may address this by engaging in casual sex with women that they would meet on only one occasion.

\footnotetext{
"What happens is that the partner is picking up cues and finds from them that they're actually being pushed away, so he won't even seek non-sexual intimacy, the kiss, the touch, the cuddles, flirtation will stop because he doesn't want to give her any clues or cues that sex might be on the cards because he is becoming avoidant of it. She will then possibly because there is no communication, internalise that as being about her, her being unattractive or that he is having sex outside the relationship, or that he is masturbating and fantasising about other partners.” (Therapist 2)
} 
"It can be that they are terrified of going near any woman and forming a relationship because they don't think that they can form a relationship." (Therapist 5)

"The most common issue I come across is avoiding sex, avoiding intimacy and then they tend to decrease in communication, they tend to talk about everything but sex and that can have a knock-on effect on relationship breakdown, that sort of thing." (Therapist 7)

\section{Involvement}

Therapists frequently commented on the role of the partner in the decision to seek treatment and the treatment process. Men often sought treatment when encouraged by partner either to improve the relationship or because the couple wished to conceive. In some instances, this presented as coercion by the partner. Some women were critical of their partner or aggressive and therapists often tried to include the partner and recognise the importance of the wider relationship. Therapists frequently commented that it was easier to evaluate treatment success if the man was in a relationship and the partner was present.

"Motivating factors that I think men come with sometimes are around partner
satisfaction, but actually that's about the partner pushing that forward and them not
actually embracing themselves, so they are coming for the wrong reason, maybe the
partner reasons are that they just want to say to the partner, well I am trying to seek help,
I've done what you told me to do, I'm getting help, lay off for a bit." (Therapist 2)
"If they don't have a partner then it's really difficult to test the treatment." (Therapist 3)
"I had a couple who did present because they wanted to conceive and he was ejaculating
prior to penetration so that was a big reason for them that made them attend." (Therapist 4)

"If there is a relationship I would more often than speak to the partner about what they are experiencing as well and how it feels for them as an individual and as a couple." (Therapist 6)

\section{Distress}

Each therapist commented on the importance of partner distress. Female partners were said to sometimes feel angry and complain that men were selfish. They distance themselves and find 
it difficult to talk about their own sexual needs. Sexual experience could influence the reaction of the partner. Female partners experience denial, fear, and can blame themselves for the PE. Therapists reported that a common fear was hurting the other person's feelings by making an issue of the PE and there were frequent explanations from partners of how much they have tried to be supportive. Though partner distress was believed to be an important issue, it was not apparent for all relationships. Women that did not enjoy penetration were often satisfied with their relationship, even if the man believed them to be distressed.

"I also think there are a category of woman who don't find their partner's PE distressing and they are the ones that often feel an obligation to have sex or that it is their duty and so they will go along with sexual activity with their partner, not enjoy it particularly and the sooner it is over the happier they are." (Therapist 1)

"Partners believing that the guy is very selfish sexually, it's all about you, it's all about your pleasure and what about me." (Therapist 2)

"I remember someone saying it was the look on her face when it happens and the feelings that it instils on him. It is bad enough for him but then it becomes the unspoken because there is that lack of communication and it becomes the big elephant in the room." (Therapist 6)

\section{Vulnerability}

\section{Self-Esteem}

Five therapists highlighted the relationship between PE and self-esteem. Therapists reported that clients judge themselves critically and have insecurity around sexual function, affecting their confidence and self-esteem. PE partners can be critical, lowering self-esteem and creating a cycle of low confidence and feelings of not being in control. This cycle can result in desperation and despair for men with PE. Successful treatment was believed to positively impact on client self-esteem. 
"Therapy makes a massive difference because there is self-confidence, self-esteem the sense of themselves as a man just develops and grows so much that it just impacts on everything. I see this in virtually every client." (Therapist 1)

"Everything changes about a man when his PE is fixed, self-esteem changes, self-worth changes." (Therapist 3)

"They tend to be low in confidence and self-esteem." (Therapist 7)

\section{Anxiety}

Therapists explained that clients with PE are affected by performance anxiety and often a more pervasive general anxiety. Therapists also stated that anxiety is inherent in some personality traits and when repeatedly triggered can be difficult to reduce. In particular, clients with PE may be impatient and have a tendency to rush treatment. Two therapists noted that for PE clients with generalised anxiety, anxiety rather than sexual function would form the initial focus, which could lead to referral.

"I think you do people a disservice trying to keep them in a sexual service when I are not best equipped to look at the fact that their anxiety is so high." (Therapist 2)

"On the whole they mostly tend to be anxious and awkward." (Therapist 4)

"I think anxiety is usually one of the main factors for people with PE because they tend to have a personality that they are anxious and that things should be done quickly." (Therapist 7)

\section{Culture}

\section{Religion and Ethnicity}

Six therapists discussed the relationship between PE and religion or ethnicity. In particular, therapists reported higher prevalence of PE in specific religious (i.e. Muslim) or ethnic (i.e. Asian) groups. Therapists highlighted a range of issues such as sexual knowledge which may contribute to increased prevalence. Religion and ethnicity impacted on the treatment process, with some clients reluctant to engage in treatment which included masturbation. One therapist also commented on the cultural barrier that may exist between therapist and client. 
Therefore therapists often considered religion and ethnicity in the assessment and treatment process.

"If you have a south Asian man, a 55 year old south Asian man, coming to see a 30 year old white British therapist, his perception that she will have had no comprehension or empathy for his cultural, religious belief systems - that can be a huge barrier." (Therapist 2)

"Some men are reluctant to do a masturbation programme, particularly in regard to religious beliefs, and that's quite common. I have a quite a few Asian men who are reluctant to participate in masturbation, even though we have discussed it with some Islamic scholars who have said in regard to treatment it's quite acceptable for them to do it if it is considered a treatment but some are still reluctant and we have to work with the other beliefs." (Therapist 4)

"I worked quite a lot with the Asian population and they tend to have a higher rate of $\mathrm{PE}$ than the rest of the PE population I work with. I don't know what that problem may be it may be because their information about sex is quite limited, due to culture or religion, I don't know." (Therapist 7)

"You get different cultures, the Asian culture especially with zero sex education which is absolutely across the board and this is what happens. They have not only, not talked about sex they are not allowed to talk about. On their wedding night they go in blind. They have no idea what they are doing and they have no idea how to do it and wonder why it doesn't work, so culture differences are hugely important in the way that clients present." (Therapist 8)

\section{Pornography}

Therapists suggested that premature-like ejaculatory issues are often the result of distorted and unrealistic sexual expectations influenced by pornography. Men do not realise that pornography is unrealistic and feel inadequate in comparison. They reported seeing more clients from this category due to a highly sexualised society with skewed beliefs about normal sexual function. As part of their intervention therapists provide psychosexual education to normalize and manage expectations. 
"They have subjected themselves to watching porn where they think that men should be able to have intercourse for hours on end and be rock hard and go from woman to woman and be ready to ejaculate again within thirty seconds, so expectations are very high." (Therapist 5)

"These people may have watched too much pornography and what they see is not real but they think this is what everybody does, can last for an hour, and so this can affect how a person perceives their sexual self and sometimes people will compare themselves, their penis size with the porn star on the pornography and that is not helpful, and that why a lot of my work is about psychosexual education." (Therapist 7)

"I think that a man's expectation of his own performance has taken a very big downturn with porn on the internet and problems all of the difficulties sexually; porn has made a lot of very insecure men out there which I think is a shame." (Therapist 8)

\section{Masculinity}

Therapists reported that men define their masculinity by their sexual prowess and function. Conversations between men often focus on sexual behaviour or achievements. Men experiencing PE could withdraw from these conversations (due to embarrassment and feelings of inadequacy) leading to ostracism. This withdrawal could be misinterpreted leading to further alienation. The impact of PE on perceived masculinity could promote men to seek treatment and often improved when their sexual function recovered.

"I think it probably is having too high an expectation as a result of conversations with other males." (Therapist 1)

"All the sexual myths that men should know about sex, men should be good at it and not having to learn and should know what women want." (Therapist 1)

"To most men their erection defines them. What they can do sexually defines them and they feel that if they can't do something it's a real, it's really bad." (Therapist 3)

\section{Diagnosis and Assessment}

\section{Referral}

Therapists frequently commented on the referral process. In particular, participants reported 
that medical practitioners are reluctant to discuss sexual issues with their patients. As a consequence, practitioners referred their patients to a therapist without appropriate assessment. The failure to assess patients was further exacerbated by a lack of practitioner understanding. Therapists reported that referrals often included a lack of appropriate information. Therefore, patients were frequently referred with the wrong diagnosis, outside the therapist's area of expertise or with a poor understanding of the treatment process. Therapists responded to these issues by discounting the information provided by medical practitioners.

\footnotetext{
“Many GP's don't like talking about sex and so the first mention of a sexual problem they would refer on and so they would not have done any questioning of whether or not the patient really had PE or not. So it would be the first mention of sex 'oh refer on'." (Therapist 1)

"What we find is the clients going in and saying I have a problem with premature ejaculation and instead of the GPs asking some fundamental questions they are just referring them straight on and then they come to me and when we look at the definition they are way outside the parameters of what we would treat and that is the main problem." (Therapist 3)

"They [client] would not be expecting to do the sort of work that I do. That has got nothing to do with me or them that is due to the GP not explaining what it is all about." (Therapist 5)

"I would say most referral diagnoses tend not to be very accurate. You can have somebody referred to you with ED and they can turn out to be PE or somebody referred with PE and they actually have ED, so really I don't tend to look at the preliminary referral diagnosis quite the same." (Therapist 7)
}

\section{Definitions}

Each therapist commented on the utility of available definitions. In particular, therapists believe formal definitions to be too rigid and were concerned that men not meeting formal criteria might be excluded from treatment. Therapists emphasised the importance of responding to client distress and individual need. In practice therapists exercised a degree of 
flexibility in their classification of PE or did not adhere to formal classifications. Four therapists did however acknowledge the value of a formal definition. For example, standardised definitions could assist discussion with clients or communication with practitioners from other disciplines.

\footnotetext{
"I think it might be helpful with some clients because if you have something written down that is quite structured it might help them to recognise that actually they have got an issue or they haven't." (Therapist 4)

"I do not use it. ... We work according to the client need; it is not about ticking boxes." (Therapist 5)

"It is great to be able to have different definitions so you as a therapist can record your work along with medical staff and other people in the same areas of work will understand which it is without a full paragraph, for example, this is situational, so yes this is helpful in a note taking environment and passing it onto other professionals." (Therapist 6)

"What worries me about definitions is that if you get too firm, too specific then what happens is someone is going to say, well that doesn't fit into the definition and you can't treat it. It worries me about a lot with services that that is what is happening in general. Definitions have become a little bit too specific. I do understand that you do need some kind of definitions but it needs to have flexibility with it." (Therapist 8)
}

\section{Discussion}

Therapists discussed the impact of PE on romantic relationships with particular emphasis on relationship intimacy, partner involvement, and partner distress. Findings are consistent with previous research documenting the association with PE and relationship avoidance (Symonds, et al., 2003), relationship difficulties (Sotomayer, 2005), relationship dissolution (Burri, Giuliano, McMahon, \& Porst, 2014), and low marital satisfaction (Ghaderi \& Rangaiah, 2012). Findings are also consistent with the increased sexual distress reported by partners of men with PE (Hartmann, Schedlowski, \& Kruger, 2005) and the perception that men focus too much attention on their own sexual performance leading their neglect of other aspects of the relationship (Phillips, 2014). The loss of intimacy and use of negative 
behaviours by some women (e.g. criticism and coercion) suggest that couples may benefit from further therapy following treatment of PE to address these relationship difficulties. Though therapists recognised the importance of partner distress, it was also reported that distress may not occur if women do not enjoy penetration. Therapists should discuss desired levels of sexual activity with both clients and partners in order to manage expectations and reduce relationship conflict. Despite these difficulties, therapists commented that evaluation of treatment success is considerably easier if the client is in a long-term sexual relationship. Therapists may therefore benefit from further support and guidance in relation to the treatment of single clients. Future research should further investigate the impact of PE on relationship dynamics and the extent to which this promotes or hinders successful treatment.

The vulnerability experienced by men with PE was clearly identified. Therapist accounts are consistent with the anxiety reported by men with PE (Dunn, Croft, \& Hackett, 1999) which may be both a cause and consequence of PE (Symonds, et al., 2003), and the poor self-esteem associated with sexual dysfunction (Intili \& Nier, 1998) and PE (Symonds, et al., 2003). Longitudinal research is required to monitor the manner in which PE influences and is influenced by anxiety and self-esteem and the extent to which treatment may improve these (Martin-Morales, Meijide, Garcia, Artes, \& Munoz, 2007). Recognition of these issues is consistent with the suggestion that supporting recovery of self-confidence and reduction of anxiety should form part of modern psychosexual therapy for PE (Montorsi, et al., 2010).

Therapists commented on the greater apparent prevalence of PE in Asian men or those from Islamic backgrounds. Their experience is consistent with the incidence of PE identified amongst men from Asian countries or Asian descent and those from Islamic backgrounds (Richardson, \& Goldmeier, 2005; Steggall, Pryce, \& Fowler, 2006). Religious or cultural beliefs may result in a belief that PE is a punishment for inappropriate sexual behaviour (i.e. 
intercourse before or outside marriage) or difficulty coping with sexual arousal when faced with sexualised imagery common to Western society (Richardson, Wood, \& Goldmeier, 2005). Therefore, education based intervention programmes targeted at Asian men may be beneficial. Future studies should consider the relationship between PE, religion, and ethnicity, and the manner in which religion and ethnicity influence the therapeutic relationship or treatment process. For example, cultural attitudes to semen loss may influence engagement in masturbation based treatment (Prakash, Kar, \& Rao, 2014) and though guidance is available for those wishing to adapt their treatment to accommodate client religious beliefs (e.g. Kellogg Spadt, et al., 2014), the extent to which this influences treatment delivery and success remains unclear.

Therapists commented on the association between pornography use and distorted sexual expectations which led to incorrect self-diagnosis of PE and distress. Previous research indicates that media exposure influences men's body dissatisfaction (Duggan \& McCreary, 2004), perceptions of masculinity (Frable, Johnson, \& Kellman, 1997), and attempts to recreate sexual acts observed (Haggstrom-Nordin, Hanson, \& Tyden, 2005). Men also believe pornography to be an important source of sexual information and advocate the inclusion of pornography into sexual education (Allen, 2006). Future research should further investigate the relationship between exposure to pornography and expectations of sexual function.

The negative impact of the traditional concept of masculinity has been recognised by both therapists in the current study and previous research (Clarke, Marks, \& Lykins, 2015). Therapists also commented however that masculinity may prompt men to seek treatment. This is consistent with previous research indicating that men may prefer to seek support and 'risk' their masculine status in order to avoid further sexual difficulties (O'Brien, Hunt, \& 
Hart, 2005) or redefine masculinity in response to sexual dysfunction (Oliffe, 2005). Those encouraging men to engage with treatment should ensure that guidance and support are consistent with traditional concepts of masculinity, particularly in those cultures emphasising masculine values (Hofstede, 1980, 2001). Further research is required in this area as multiple versions of masculinity exist (Connell, 1995).

Therapists identified a number of issues relating to the referral process. General practitioners were believed to be unwilling to discuss sexual health and function with patients, leading to inadequate assessment and referral. These reports are consistent with previous research indicating that though patients believe sexual issues to be an appropriate subject for discussion, practitioners rarely discuss sexual dysfunction with patients or record sexual difficulties (Read, King, \& Watson, 1997; Ribeiro, et al., 2014). This may reflect a lack of sexual dysfunction training or guidance and a lack of time (Solursh, et al., 2003) which may lead some general practitioners to avoid these discussions (Gott, Galena, Hinchliff, \& Elsford, 2004). Indeed practitioners have expressed a need for further training and experience in the area of sexual dysfunction (Alarcao, et al., 2012). This is essential if (as proposed) primary care providers are to support diagnosis of PE (Althof, et al., 2010). Greater communication between therapists and medical practitioners is required to support the use of formal guidelines and ensure that the client experience is not impacted by ineffective or inaccurate referral. Further research is required to establish those factors which may influence general practitioner referral (Hackl, Hummer, \& Pruckner, 2015) and the manner in which interventions may enhance general communication with patients or therapists.

Historically, the lack of a universally agreed definition has hindered accurate diagnosis of PE (Carson \& Gunn, 2006). The recent DSM-5 definition (APA, 2013) provides a clear and detailed description of PE which can be used to enhance PE assessment and diagnosis. 
Further classification of PE type as advised in the DSM-5 may also guide the type of treatment selected (Rowland, et al., 2010). Therapists were however critical of the definition, which was believed to be too rigid, and the general use of definitions, which therapists feared could result in the denial of treatment to men that did not meet formal criteria. The utility of the definition may therefore be limited. A number of therapists did recognise the benefits afforded by a universal definition which could enhance communication with clients or other practitioners. Additional information assessing the effectiveness of this process is required. Therefore, the extent to which formal definitions guided practice and the diagnosis or treatment of PE varied, with important implications for comparisons between client groups. Further research is recommended to investigate the relationship between therapist perceptions of formal definitions, PE practice, and treatment outcomes.

These findings provide an important insight into the lived experience of therapists supporting men with PE. Consistent with Interpretive Phenomenological Analysis, which focuses on the acquisition of rich detailed information, a small sample was recruited which limits the representativeness of the sample and generalisability of the findings. Findings should therefore be interpreted with caution. Future research should investigate the extent to which therapist experiences are influenced by factors such as therapist sex, therapeutic approach, and culture; and whether these impact on the client experience or treatment outcomes. Comparisons between professional groups (Wylie, 2006) or forms of therapy (Libman, Fichten, \& Brender, 1985) may be particularly beneficial.

To conclude, interviews were conducted with eight Psychosexual Therapists and subjected to Interpretive Phenomenological Analysis. Four superordinate themes emerged from the analysis. These were Romantic Relationships, Vulnerability, Culture and Diagnosis and Assessment. The Romantic Relationships theme contained three sub-themes: Intimacy; 
Involvement; and Distress. The Vulnerability theme contained two sub-themes: Self-Esteem and Anxiety. The Culture theme contained three sub-themes: Religion and Ethnicity; Pornography; and Masculinity. The Diagnosis and Assessment theme contained two subthemes: Referral and Definition. Findings have important implications for the diagnosis and treatment of PE. Future research should investigate therapist perceptions further, including those factors which impact on engagement with clients and PE treatment success.

\section{References}

Ackerman, S. J., \& Hilsenroth, M. J. (2003). A review of therapist characteristics and techniques positively impacting the therapeutic alliance. Clinical Psychology Review, 23, 1-33. doi:10.1016/S0272-7358(02)00146-0

Allen, L. (2006). "Looking at the real thing": Young men, pornography, and sexuality education. Discourse: Studies in the Cultural Politics of Education, 27, 69-83. doi: $10.1080 / 01596300500510302$

Alarcao, V., Ribeiro, S., Miranda, F. L., Carreira, M., Dias, T., Garcia e Costa, J., \& GalvaoTeles, A. (2012). General Practitioners' knowledge, attitudes, beliefs, and practices in the management of Sexual Dysfunction - Results of the Portuguese SEXOS study. Journal of Sexual Medicine, 9, 2508-2515. doi: 10.1111/j.1743-6109.2012.02870

Althof, S. E., Abdo, C. H. N., Dean, J., Hackett, G., McCabe, M., McMahon, C. G.,...Tan, H. M. (2010). International Society for Sexual Medicine's guidelines for the diagnosis and treatment of Premature Ejaculation. Journal of Sexual Medicine, 7, 2947-2969. doi: 10.1111/j.1743-6109.2010.01975

American Psychiatric Association (2013). Diagnostic and statistical manual of mental disorders DSM-5. Washington: American Psychiatric Association.

Arvinen-Barrow, M., Penny, G., Hemmings, B., \& Corr, S. (2010). UK chartered physiotherapists' personal experiences in using psychological interventions with injured athletes: An Interpretative Phenomenological Analysis. Psychology of Sport and Exercise, 11, 58-66. doi: 10.1016/j.psychsport.2009.05.004

Burri, A., Giuliano, F., McMahon, C., \& Porst, H. (2014). Female partner's perception of Premature Ejaculation and its impact on relationship breakups, relationship quality, 
and sexual satisfaction. Journal of Sexual Medicine, 11, 2243-2255. doi: $10.1111 /$ jsm. 12551

Carson, C., \& Gunn, K. (2006). Premature ejaculation: Definition and prevalence. International Journal of Impotence Research, 18, S5-S13. doi: 10.1038/sj.ijir.3901507

Clarke, M. J., Marks, A. D. G., \& Lykins, A. D. (2015). Effect of normative masculinity on males' dysfunctional sexual beliefs, sexual attitudes, and perceptions of sexual functioning. Journal of Sex Research, 52, 327-337. doi: $10.1080 / 00224499.2013 .860072$

Connell, R. (1995). Masculinities. Berkeley, CA: University of California Press.

Corretti, G., Pierucci, S., De Scisciolo, M., \& Nisita, C. (2006). Comorbidity between social phobia and premature ejaculation: Study on 242 males affected by sexual disorders. Journal of Sex and Marital Therapy, 32, 183-187. doi: 10.1080/00926230500442342

Corty, E. W., \& Guardiani, J. M. (2008). Canadian and American sex therapists' perceptions of normal and abnormal ejaculatory latencies: How long should intercourse last? The Journal of Sexual Medicine, 5, 1251-1256. doi: 10.1111/j.1743-6109.2008.00797.x

Duggan, S. J., \& McCreary, D. R. (2004). Body image, eating disorders, and the drive for muscularity in gay and heterosexual men. Journal of Homosexuality, 47, 45-58. doi: 10.1300/J082v47n03_03

Dunn, K. M., Croft, P. R., \& Hackett, G. I. (1999). Association of sexual problems with social, psychological, and physical problems in men and women. A cross-sectional population survey. Journal of Epidemiology and Community Health, 53, 144-148.

Frable, D. E. S., Johnson, A. E., \& Kellman, H. (1997). Seeing masculine men, sexy women, and gender differences: Exposure to pornography and cognitive constructions of gender. Journal of Personality, 65, 311-355. doi: 10.1111/j.14676494.1997.tb00957.x

Ghaderi, A. R., \& Rangaiah, B. (2012). Premature ejaculation and its influence on marital satisfaction. Journal of Psychosomatic Research, 7, 195-202.

Gott, M., Galena, E., Hinchliff, S., Elford, H. (2004). "Opening a can of worms”: GP and practice nurse barriers to talking about sexual health in primary care. Family Practice, 21, 528-536. doi: 10.1093/fampra/cmh509

Hackl, F., Hummer, M., \& Pruckner, G. J. (2015). Old boys' network in general practitioners' referral behavior? Journal of Health Economics, 43, 56-73. doi:10.1016/j.jhealeco.2015.06.005 
Haggstrom-Nordin, E., Hanson, U., \& Tyden, T. (2005). Associations between pornography consumption and sexual practices among adolescents in Sweden. International Journal of STD and AIDS, 16, 102-107. doi: 10.1258/0956462053057512

Hall, M. (2002). Not tonight dear, I'm deconstructing a headache. Women \& Therapy, 24, 161-172. doi: 10.1300/JO15v24n01 18

Hartmann, U., Schedlowski, M., \& Kruger, T. H. (2005). Cognitive and partner-related factors in rapid ejaculation: Differences between dysfunctional and functional men. World Journal of Urology, 23, 93-101.

Hofstede, G. H. (1980). Culture's consequences: International Differences in work-related values. Beverly Hills, CA: Sage.

Hofstede, G. H. (2001). Culture's consequences: Comparing values, behaviors, institutions and organizations across nations ( $2^{\text {nd }}$ Ed). Thousand Oaks, CA: Sage.

Intili, H., \& Nier, D. (1998). Self-esteem and depression in men who present with erectile dysfunction. Urologic Nursing, 18, 185-188.

Kellogg Spadt, S., Rosenbaum, T. Y., Dweck, A., Millheiser, L., Pillai-Friedman, S., \& Krychman, M. (2014). Sexual health and religion: A primer for the sexual health clinician. Journal of Sexual Medicine, 11, 1606-1619. doi: 10.1111/jsm.12593

Kirschneck, M., Kirchberger, I., Amann, E., \& Cieza, A. (2011). Validation of the comprehensive ICF core set for low back pain: The perspective of physical therapists. Manual Therapy, 16, 364-372. doi:10.1016/j.math.2010.12.011

Libman, E., Fichten, C. S., \& Brender, W. (1985). The role of therapeutic format in the treatment of sexual dysfunction: A review. Clinical Psychology Review, 5, 103-117.

Mackereth, P., Parekh, J., \& Donald, G. (2012). Providing therapies to the opposite sex: Views of complementary therapists working in clinical and private practice settings. Complementary Therapies in Clinical Practice, 18, 154-158. doi:10.1016/j.ctcp.2012.05.001

Maguire, R., Stoddart, K., Flowers, P., McPhelim, J., \& Kearney, N. (2014). An Interpretative Phenomenological Analysis of the lived experience of multiple concurrent symptoms in patients with lung cancer: A contribution to the study of symptom clusters. European Journal of Oncology Nursing, 18, 310-315. doi: 10.1016/j.ejon.2014.02.004

Martin-Morales, A., Meijide, F., Garcia, N., Artes, M., \& Munoz, A. (2007). Efficacy of Vardenafil and influence on self-esteem and self-confidence in patients with severe 
erectile dysfunction. The Journal of Sexual Medicine, 4, 440-447. doi: $10.1111 / \mathrm{j} .1743-6109.2006 .00426 . x$

McCaffrey, T. (2013). Music therapists' experience of self in clinical improvisation in music therapy: A phenomenological investigation. The Arts in Psychotherapy, 40, 306-311. doi: 10.1016/j.aip.2013.05.018

Montorsi, F., Adaikan, G., Becher, E., Giuliano,F.,Khoury, S., Lue, T. F.,...Wasserman, M. (2010). Summary of the recommendations on sexual dysfunctions in men. Journal of Sexual Medicine, 7, 3572-3588. doi: 10.1111/.1743-6109.2010.02062

O’Brien, R., Hunt, K., \& Hart, G. (2005). 'It's caveman stuff, but that is to a certain extent how guys still operate': Men's accounts of masculinity and help seeking. Social Science \& Medicine, 61, 503-516.

Oliffe, J. (2005). Constructions of masculinity following prostatectomy-induced impotence. Social Science \& Medicine, 60, 2249-2259. doi:10.1016/j.socscimed.2004.10.016 Phillips, R. (2014). Premature ejaculation and female sexual satisfaction. Nature Reviews Urology, 11, 304. doi: 10.1038/nrurol.2014.114

Porst, H., Montorsi, F., Rosen, R. C., Gaynor, L., Grupe, S., \& Alexander, J. (2007). The Premature Ejaculation Prevalence and Attitudes (PEPA) survey: Prevalence, comorbidities, and professional help-seeking. European Urology, 51, 816-824. doi:10.1016/j.eururo.2006.07.004

Prakash, O., Kar, S. K., \& Rao, T. S. S. (2014). Indian story on semen loss and related Dhat syndrome. Indian Journal of Psychiatry, 56, 377-382. doi: 10.4103/00195545.146532

Read, S., King, M., \& Watson, J. (1997). Sexual dysfunction in primary medical care: Prevalence, characteristics and detection by the general practitioner. Journal of Public Health Medicine, 19, 387-391.

Ribeiro, S., Alarcao, V., Simoes, R., Miranda, F. L., Carreira, M., \& Galvao-Teles, A. (2014). General Practitioners' procedures for sexual history taking and treating sexual dysfunction in primary care. Journal of Sexual Medicine, 11, 386-393. doi: $10.1111 /$ jsm. 12395

Richardson, D., \& Goldmeier, D. (2005). Premature ejaculation: Does country of origin tell us anything about etiology? The Journal of Sexual Medicine, 2, 508-512. doi: 10.1111/j.1743-6109.2005.00074.x 
Richardson, D., Wood, K., \& Goldmeier, D. (2005). A qualitative pilot study of Islamic men with lifelong premature (rapid) ejaculation. Journal of Sexual Medicine, 3, 337-343. doi: 10.1111/j.1743-6109.2005.00175.x

Rosen, R. C., \& Althof, S. (2008). Impact of premature ejaculation: The psychological, quality of life, and sexual relationship consequences. Journal of Sexual Medicine, 5, 1296-1307. doi: 10.1111/j.1743-6109.2008.00825

Rowland, D. L., McMahon, C. G., Abdo, C., Chen, J., Jannini, E., Waldinger, M. D., \& Ahn, T. Y. (2010). Disorders of orgasm and ejaculation in men. Journal of Sexual Medicine, 7, 1668-1686. doi: 10.1111/j.1743-6109.2010.01782

Rowland, D. L., Patrick, D. L., Rothman, M., Gagnon, D. D. (2007). The psychological burden of premature ejaculation. Journal of Urology, 177, 1065-1070. doi:10.1016/j.juro.2006.10.025

Rybovic, M., Halkett, G. K., Banati, R. B., \& Cox, J. (2008). Radiation therapists' perceptions of the minimum level of experience required to perform portal image analysis. Radiography, 14, 294-300. doi:10.1016/j.radi.2007.07.004

Smith, J. A. (1995). Semi-structured interviewing and qualitative analysis. In J. A. Smith, R. Harre, \& L. van Langenhove (Eds.). Rethinking methods in psychology. London: Sage.

Smith, J. A. (1996). Beyond the divide between cognition and discourse: Using Interpretative Phenomenological Analysis in health psychology. Psychology and Health, 11, 261271.

Smith, J. A., Flowers, P., \& Larkin, M. (2009). Interpretive phenomenological analysis: Theory, method and research. London: Sage.

Solursh, D. S., Ernst, J. L., Lewis, R. W., Prisant, L. M., Mills, T. M., Solursh, L. P., Jarvis, R. G., \& Salazar, W. H. (2003). The human sexuality education of physicians in North American medical schools. International Journal of Impotence Research, 15, S41-S45.

Sotomayor, M. (2005). The burden of premature ejaculation: The patient's perspective. Journal of Sexual Medicine, 2, 110-114. doi: 10.1111/j.1743-6109.2005.20371.x

Steggall, M. J., Pryce, A., \& Fowler, C. G. (2006). Is ethnicity and religion an aetiological factor in men with rapid ejaculation? Sexual and Relationship Therapy, 21, 429-437. doi: 10.1080/14681990600667371 
Symonds, T., Roblin, D., Hart, K., \& Althof, S. (2003). How does premature ejaculation impact a man's life? Journal of Sex and Martial Therapy, 29, 361-370. doi: $10.1080 / 00926230390224738$

Wylie, K. R. (2006). Professional and clinical differences amongst clinicians dealing with sexual problems in the United Kingdom. Sexologies, 15, 30-34. doi:

10.1016/j.sexol.2205.11.001 
Table 1: Participant Characteristics

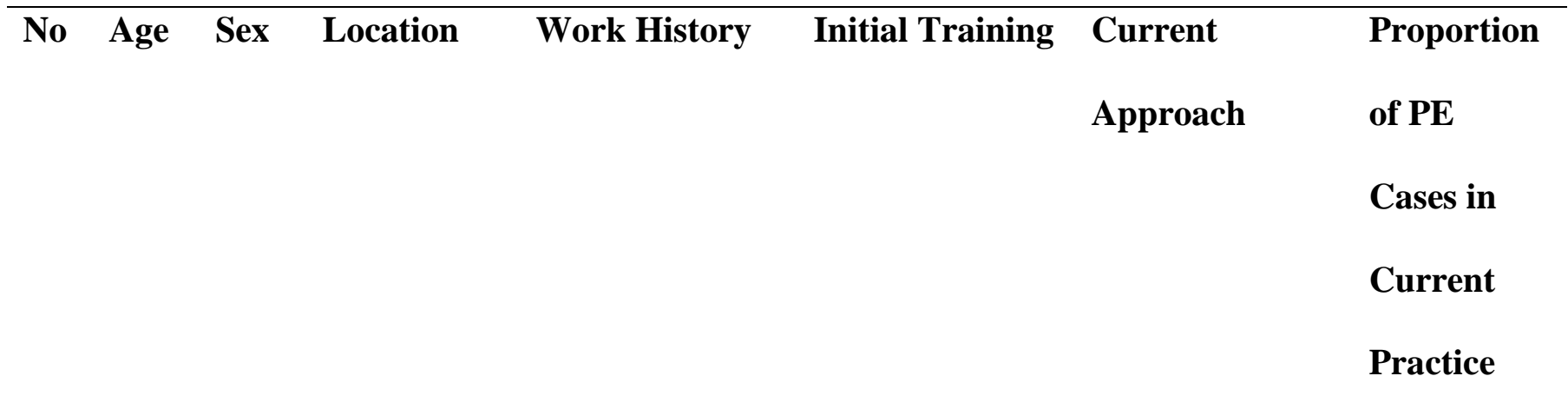

\begin{tabular}{llll}
\hline 1 & 60 & F & Private
\end{tabular}

27 yrs as a

Couple

Therapist \& 20

yrs as a

Psychosexual

Therapist

$\begin{array}{lllll}2 & 52 & \mathrm{~F} & \text { Private } \& \quad 15 \mathrm{yrs} \text { as a }\end{array}$

NHS

Psychosexual

Therapist

$3 \quad 48 \quad F \quad$ Private

10 yrs as a

Psychotherapist

$\& 4$ yrs as a

Psychosexual

Therapist

Eclectic -

Eclectic -

$20 \%$

Integrated

Integrated

Systemic,

Psychodynamic

\& CBT

$20 \%$ 
$\begin{array}{llll}5 & 63 & F & \text { Private }\end{array}$

18 yrs as a

Integrative

CBT

$5-10 \%$

Psychosexual

Therapist

6

$45 \quad$ F $\quad$ Private \& $\quad 11$ yrs as a

Psychodynamic, $\quad$ CBT \&

$20 \%$

NHS

Couple

Systemic, \&

Systemic

Therapist \& 6

CBT

yrs as a

Psychosexual

Therapist

7
M
Private \&
16 yrs as a

47

Person-Centred Eclectic with

$20 \%$

NHS

Psychotherapist

Person-Centred,

$\& 4$ years as a

Systemic,

Psychosexual

Psychodynamic,

Therapist

$\&$ CBT

8

Private \&

14 years as a

Psychodynamic

$25 \%$

NHS Couple

Therapist \& 12

yrs as a

Psychosexual

Therapist 
Table 2: Superordinate and Sub-Themes

\begin{tabular}{ll}
\hline Superordinate Theme & Sub-Theme \\
\hline Romantic Relationships & Intimacy \\
& Involvement \\
& Distress \\
Vulnerability & Self-Esteem \\
& Anxiety \\
Culture & Religion and Ethnicity \\
& Pornography \\
Diagnosis and Assessment & Masculinity \\
& Referral \\
\hline
\end{tabular}

